\title{
OXYGEN TENSION OF TISSUES BY THE POLAROGRAPHIC METHOD. IV. SKIN OXYGEN TENSION VS. ARTERIAL OXYGEN SATURATION; RELA- TIONSHIP TO OXYHEMOGLOBIN DISSOCIATION CURVE
}

\author{
BY RAYMOND PENNEYS 1 \\ (From the Peripheral Vascular Section, Robinette Foundation, Medical Clinic, Hospital of the \\ University of Pennsylvania, Philadelphia, $\mathrm{Pa}$.)
}

(Submitted for publication July 13, 1951 ; accepted November 20, 1951)

\section{INTRODUCTION}

The relationship between the oxygen saturation and oxygen tension of the blood has, up to the present time, been studied by methods requiring in vitro analysis. These methods, such as the. Van Slyke-Neill manometric (1) and Krogh equilibration (2) techniques, require considerable time and skill and give only discontinuous measurements. This report describes an indirect study of this relationship by simple instrumental methods which supply immediate and continuous information in vivo. They consist of the oxygen electrode (3), which can be used to measure polarographically the oxygen tension in the intact human skin (4), and the oximeter $(5,6)$, which measures the arterial blood oxygen saturation in the intact human ear.

In this paper are described the changes in the oxygen tension of skin warmed by a rapid circulation, brought on by altering the blood arterial oxygen saturation in normal human subjects (7). The correlation of skin oxygen tension and blood arterial saturation and its relationship to the oxyhemoglobin dissociation curve are demonstrated.

\section{METHOD}

The method of producing alterations of the blood arterial oxygen saturation has been described previously (7). It consists of administering constantly varying mixtures of pure oxygen and pure nitrogen through an anesthesia machine to obtain and maintain the desired level of the arterial oxygen saturation, as measured by the Millikan oximeter.

The subject, shortly after eating his regular meal, lay quietly for approximately 45 minutes, breathing air, before inhaling the gas mixtures. Skin temperature readings were taken, the oximeter was attached to the ear,

1 Instructor in Medicine, University of Pennsylvania School of Medicine. The major portion of this work was done during the tenure of a Post-Doctorate Fellowship, United States Public Health Service. Support was also received from the Life Insurance Medical Research Fund. and the oxygen electrodes were inserted in the skin. "Ear-thickness" readings were taken on the oximeter until stable and the "saturation" was then set at " 100 " while the subject breathed pure oxygen $(5,6)$. Oxygen tension readings were taken at frequent intervals until they became stable. A low or high oxygen mixture was then inhaled. After the inhalation of this gas mixture, the subject was again allowed to breathe room air. In some experiments the arterial saturation and the oxygen tension changes during this period of recovery were noted.

The saturations studied ranged from 67 to $100 \%$. Any change from one level of saturation to another was made gradually, and the saturation was kept constant at the desired level for several minutes. While the saturation was held constant, oxygen tension readings were taken, in most experiments until they too became constant (solid circles, Figure 1). In some of the experiments, where the effect of reduced arterial oxygen saturation was being studied, repeated oxygen tension readings were not taken (open circles, Figure 1) at each level of saturation in order not to prolong unduly the total period of hypoxia. In these instances readings were taken only after the desired saturation had been maintained for a period of time found, from the other experiments, to be sufficient for the skin oxygen to come into equilibrium with the blood arterial oxygen.

The oxygen electrode, method of taking readings, and corrections for temperature variations have been described by Montgomery and Horwitz (4). In the present experiments, however, the electrodes were uncalibrated so that only relative changes were measured. The reading obtained while the subject was breathing air was arbitrarily designated at 100 . The electrodes were sterilized by boiling and their tips were kept in sterile saline solution when not in use. The electrodes were inserted in the skin of the outer aspect of the arm of each subject. Eight different electrodes were used and, in most of the experiments, the same set of five electrodes.

The first three experiments, in which only two electrodes were used, showed that the oxygen tension percentages of the two electrodes, at a given arterial saturation, differed significantly. To minimize the effect of these differences, from three to five electrodes were used in all subsequent experiments, the oxygen tension (Figure 1) being taken as the average of values obtained by the several electrodes. 
Skin temperature was recorded continuously throughout each experiment by thermocouples (8) placed on different areas of the skin near the various electrodes. In order to maintain a rapid circulation through the skin of the arm it was occasionally necessary to apply heat elsewhere to the body. The skin temperatures were kept between 30 and $34^{\circ} \mathrm{C}$ except in two instances where the temperature of the skin could not be raised above $29^{\circ} \mathrm{C}$. The room temperature of all experiments averaged $26^{\circ} \mathrm{C}$. Neither skin nor room temperature varied more than one degree throughout any one experiment.

Fourteen normal, white adults, ages 18 to 46 , were used as subjects; all but three were males. A total of 31 experiments was performed.

\section{OBSERVATIONS}

The effect of decreasing or increasing the blood arterial oxygen saturation on the oxygen tension of the skin is shown in Figure 1, where the results of the 31 experiments are plotted. In each experiment the oxygen tension changed in the same direction as the arterial saturation.

One can see in Figure 1 striking differences in the amount of change in oxygen tension, per unit change in arterial saturation, at different levels of arterial saturation. In lowering the saturation from 75 to $70 \%$, for instance, the tension was decreased by only $4 \%$. In lowering the saturation from 95 to $90 \%$, the tension, on the other hand, was decreased by $20 \%$. The large changes in tension at the high arterial saturations were especially apparent in several instances (not included in Figure 1) where the arterial saturation was progressively increased between 96 and $99 \%$ saturation by small increments ranging from $1 / 4$ to $1 \%$ arterial saturation. These small changes in saturation, regardless of how gradually they were made, always produced considerable increases in oxygen tension.

Several other observations on the relationship between the arterial oxygen saturation and skin oxygen tension were made:

1. The recovery of the oxygen tension and the arterial oxygen saturation: Observations on the rate of recovery of the arterial saturation and the skin oxygen tension were made when the subject was allowed to breathe room air immediately upon the termination of some of the experiments. After low oxygen inhalation (two experiments), the arterial saturation fully regained its normal value in approximately five minutes while the skin oxygen tension required about 10 minutes. After high oxygen (six experiments), the saturation also returned to normal in approximately five minutes and the tension again required twice as much time.

The final recovery value of the arterial saturation, noted in most instances after the subject had

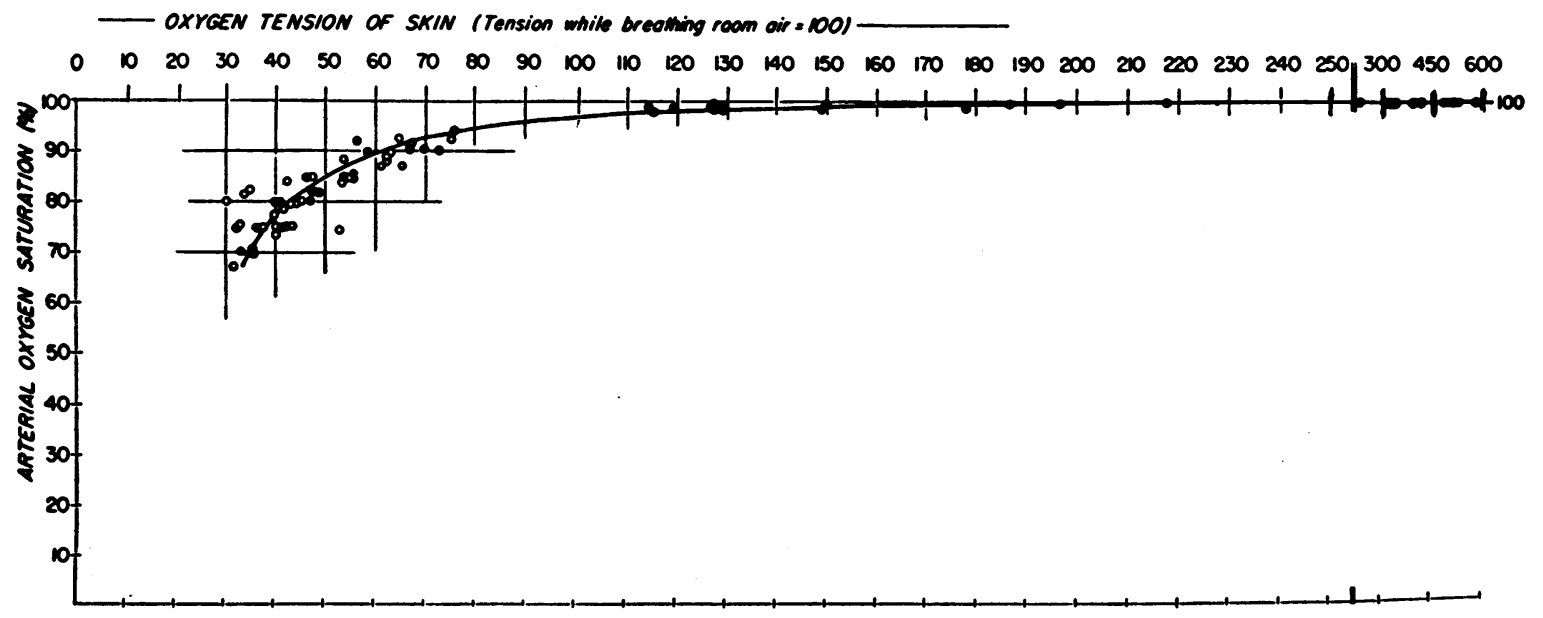

Fig. 1. Skin Oxygen Tension Versus Arterial Oxygen Saturation

The skin oxygen tension of each of the points was obtained by averaging the values of several electrodes used simultaneously.

- Skin oxygen tension in equilibrium with arterial oxygen as obtained by repeat tension readings at each level of arterial saturation (see Method).

O Skin oxygen tension in equilibrium with arterial oxygen as obtained by single tension readings at each level of arterial saturation after the saturation had been maintained constant for several minutes (see Method). 


\section{TABLE I}

Oxygen tension of the skin, in galvanometer units, while subject is breathing air

Values "For each experiment" are average of all electrodes used in each experiment. One very high reading, 88 , obtained with electrode "B," was not included. Readings were obtained after electrodes had been in the skin for approximately 30 minutes and were stable.

\begin{tabular}{l|c|c|c|c|c|c}
\hline \hline & $\begin{array}{c}\text { For each } \\
\text { experi- } \\
\text { ment }\end{array}$ & \multicolumn{4}{|c}{ For each electrode } \\
\cline { 2 - 7 } & $\mathrm{A}$ & $\mathrm{B}$ & $\mathrm{C}$ & $\mathrm{D}$ & $\mathrm{E}$ \\
\hline $\begin{array}{l}\text { Oxygen tension } \\
\text { Average } \\
\text { Stand dev. } \\
\text { Range }\end{array}$ & 20 & 19.2 & 25.3 & 18.2 & 17.2 & 16.8 \\
\hline No. experiments & 13.6 & 3.2 & 9.4 & 6.1 & 8.7 & 5.6 \\
\hline
\end{tabular}

been breathing room air for approximately 15 minutes, was about the same as the initial reading on room air. The oxygen tension on recovery from breathing low oxygen averaged $91 \%$ (range 73 to $108 \%$ ) of the initial room air reading, whereas it was $106 \%$ (range 86 to $138 \%$ ) after the inhalation of pure oxygen.

In a few instances a droplet of blood was found when the electrodes were removed. This had no apparent effect on the readings, unlike the experience of Montgomery and Horwitz (4).

2. Effect of carbon dioxide: In a single experiment, in which the arterial saturation was maintained at $80 \%, 2.5 \% \mathrm{CO}_{2}(9)$, included in the gas mixture for a period of 10 minutes, caused no measurable change in the oxygen tension.

3. Skin oxygen tension in galvanometer units while breathing air: It was noted throughout these experiments that the oxygen tension reading of skin, warmed by a rapid circulation, was fairly consistent while the subjects were breathing air. The readings for the five electrodes used most frequently were analyzed and are listed in Table I. The average value of each of these five electrodes ranged between 16.8 and 25.3 galvanometer units.

\section{DISCUSSION}

Under the conditions of these experiments, in which an attempt was made to maintain a rapid cutaneous circulation, the oxygen tension of the skin, measured polarographically by the oxygen tension electrode, changed consistently with the blood arterial oxygen saturation, as measured by the Millikan oximeter.
The quantitative relationship between the tension, expressed in percent of initial value while breathing air, and the saturation may be represented by a curve (Figure 1) which, between $67 \%$ arterial saturation and the upper limit of the oxyhemoglobin dissociation curves obtained by gasometric analysis (10-12), has the characteristically changing slope of these latter curves. At the higher saturations in the present experiments, the relationship is closely similar to that reported by direct polarographic measurements on the arterial blood (13). The oxygen tension of the skin having a fast circulation thus bears the same type of relationship to the arterial oxygen saturation as does the arterial oxygen tension.

Some technical and physiological limitations of these experiments may be emphasized: 1 . The observations extend down to only $67 \%$ arterial saturation. Hypoxemic patients may offer an opportunity to study the curve at lower levels. 2 . Certain limitations are inherent in the Millikan oximeter $(5,6,14)$ and in the stationary platinum oxygen electrode (4). When the arterial oxygen saturation was increased, or decreased, to any given level, there was a considerable difference between tension changes of individual electrodes. These differences are unexplained and demonstrate the importance of using several electrodes in determining the degree of any change. 3. An attempt was made to maintain a cutaneous circulation fast enough (as indicated by differences between skin temperature and room temperature) to supply oxygen in excess of metabolic needs. Variations in cutaneous circulation or oxygen metabolism are expected to contribute some error (4). 4. Two important physiological factors, the $\mathrm{pH}(6)$ and the temperature $(15,16)$ of the blood and tissues, were not completely controlled or measured. It is of interest to note that the scatter of observations in Figure 1 closely resembles the scatter of points of the conventional dissociation curve having alterations of the blood $\mathrm{pH}$ over the range 7.2 to $7.6(6)$. Considering the above, the curve may prove to be more useful if some of these variables are better controlled.

By the simultaneous use of the oximeter and oxygen electrode, clinical disturbances related to the oxygen dissociation of the blood may be more readily laid open to investigation. The effect of various metabolic and hypoxemic states on the 
oxyhemoglobin dissociation of blood may be studied at the bedside of critically ill patients without discomfort or the loss of blood.

Two other observations on the relationship between the arterial oxygen saturation and the oxygen tension of the skin were made: 1 . The oxygen tension of the skin, after the inhalation of low or high oxygen mixtures, required several minutes longer than the arterial saturation to return fully to its original value. This agrees with other studies made on the viscera of animals (17). 2 . The consistency of all the oxygen tension galvanometric readings on our subjects, while they were breathing air (Table I), suggests that the uncalibrated oxygen electrode may be used to establish an "oxygen tension reading," in galvanometric units, of skin with a rapid circulation in normal subjects. Such an electrode may then be useful in studying changes in oxygen tension in skin and their implications regarding the central and peripheral circulation.

\section{SUM MARY}

1. The blood arterial oxygen saturation of normal human subjects was altered by the inhalation of a gas of varying oxygen concentration to give readings between 67 and $100 \%$, as measured by the Millikan oximeter. Simultaneously the oxygen tension of the skin, having a reasonably rapid circulation, was measured by several oxygen electrodes.

2. The oxygen tension changed in the same direction as did the arterial saturation. When the tension, expressed in percent of the reading obtained while breathing air, was plotted against the arterial oxygen saturation, a curve was obtained (Figure 1) with the characteristically changing slope of the conventional oxyhemoglobin dissociation curves of blood.

3. After the inhalation of low or high oxygen mixtures, the skin oxygen tension returned more slowly to initial values than did the arterial oxygen saturation.

4. Unexplained differences in the tension changes between the individual electrodes made the simultaneous use of several electrodes advisable.

5. Oxygen tension galvanometric readings obtained while breathing air were fairly consistent, suggesting that the uncalibrated oxygen electrode may be used to establish an "oxygen tension reading" of skin with a rapid circulation in normal subjects.

\section{ACKNOWLEDGMENTS}

The author would like to express his appreciation to Dr. Hugh Montgomery for his very helpful advice and to Miss Ann Sayen for her expert technical assistance.

\section{REFERENCES}

1. Van Slyke, D. D., and Neill, J. M., The determination of gases in blood and other solutions by vacuum extraction and manometric measurement. I. J. Biol. Chem., 1924, 61, 523.

2. Krogh, A., Some new methods for tonometric determination of gas-tensions in fluids. Skandinav. arch. Physiol., 1908, 20, 259.

3. Davies, P. W., and Brink, F., Jr., Microelectrodes for measuring local oxygen tension in animal tissues. Rev. Scient. Instruments, 1942, 13, 524.

4. Montgomery, H., and Horwitz, O., Oxygen tension of tissues by the polarographic method. I. Introduction: oxygen tension and blood flow of the skin of human extremities. J. Clin. Invest., 1950, 29, 1120.

5. Millikan, G. A., The oximeter, an instrument for measuring continuously the oxygen saturation of arterial blood in man. Rev. Scient. Instruments, 1942, 13, 434.

6. Comroe, J. H., Jr., Methods in Medical Research. The Year Book Publishers, 1950, Vol. 2.

7. Penneys, R., Thomas, C. B., and McLean, R. L., A constant degree of anoxemia obtained by the administration of a gas of variable oxygen concentration. Bull. Johns Hopkins Hosp., 1949, 84, 430.

8. Brown Electronik Potentiometer. The Brown Instruments Division of Minneapolis-Honeywell Regulator Company, Philadelphia, Pa.

9. Penneys, R., A method of administering carbon dioxide at a constant degree of induced anoxemia and its cardiovascular effects. Bull. Johns Hopkins Hosp., 1950, 86, 107.

10. Barcroft, J., The combinations of haemoglobin with oxygen and with carbon monoxide. II. Biochem. J., 1913, 7, 481.

11. Bock, A. V., Field, H., Jr., and Adair, G. S., The oxygen and carbon dioxide dissociation curves of human blood. J. Biol. Chem., 1924, 59, 353.

12. Dill, D. B., as quoted by Riley, R. L., Lilienthal, J. L., Jr., Proemmel, D. D., and Franke, R. E., The relationships of oxygen, carbon dioxide, and hemoglobin in the blood of man: oxyhemoglobin dissociation under various physiological conditions. J. Clin. Invest., 1946, 25, 139.

13. Nahas, G. G., Morgan, E. H., and Wood, E. H., Oxygen dissociation curve in arterial blood of man breathing high oxygen mixtures using polaro- 
graphic and photometric methods for whole blood. Am. J. Physiol., 1950, 163, 737.

14. Penneys, R., The correlation between the ear thickness and saturation readings of the Millikan oximeter. Bull. Johns Hopkins Hosp., 1950, 87, 215.

15. Brown, W. E. L., and Hill, A. V., The oxygen-dissociation curve of blood and its thermodynamical basis. Proc. Roy. Soc., London, 1923, Ser. B. 94, 297.
16. Horwitz, O., Peirce, G., and Montgomery, H., Oxygen tension of tissues by the polarographic method. III. The effect of local heat on the oxygen tension of the skin of extremities. Circulation, 1951, 4, 111.

17. Behrmann, V. G., Griest, W. D., Mangun, G. H., and Hartman, F. W., Comparison of arterial $\mathrm{O}_{2}$ saturation with tissue $\mathrm{O}_{2}$ levels. Federation Proc., 1950, 9, 10. 